\title{
NEAR-INSTANTANEOUSLY ADAPTIVE DECISION FEEDBACK EQUALIZED CODED MODULATION FOR WIRELESS VIDEO TELEPHONY
}

\author{
P. Cherriman, S. X. Ng, L. Hanzo \\ Dept. of ECS, Univ. of Southampton, SO17 1BJ, UK. \\ Tel: +44-1703-593 125, Fax: +44-1703-594 508 \\ Email:lhdecs.soton.ac.uk, http://www-mobile.ecs.soton.ac.uk
}

\begin{abstract}
Decision Feedback Equaliation (DFE) aided wideband Burstby-Burst (BbB) Adaptive Trellis Coded Modulation (TCM), Turbo Trellis Coded Modulation (TTCM) and Bit-Interleaved Coded Modulation (BICM) are proposed and characterised in performance terms over the COST 207 Typical Urban (TU) wideband fading channel. These schemes are evaluated using a practical modem mode switching regime in the context of wireless video telephony.
\end{abstract}

\section{INTRODUCTION}

The radio spectrum is a scarce resource. Therefore, one of the most important objectives in the design of a digital cellular system is the efficient exploitation of the available spectrum in order to accommodate the ever-increasing traffic demands. Trellis Coded Modulation (TCM) [1], which is based on combining the functions of coding and modulation, is a bandwidth efficient scheme that has been widely recognized as an excellent error control technique suitable for applications in mobile communications [2,3]. Turbo Trellis Coded Modulation (TTCM) [4] is a more recent channel coding scheme that has a structure similar to that of the family of power efficient binary turbo codes [5], but employs TCM codes as component codes. TCM and TTCM schemes invoked Set-Partitioning (SP) based modulated symbol labeling, in order to achieve an increased Euclidean distance between the unprotected bits of the constellation. As a consequence, parallel trellis transitions can be associated with the unprotected data bits. This reduced the decoding complexity. In the TCM and TTCM schemes investigated random symbol interleaver arrangements were utilised for both the turbo interleaver and the channel interleaver.

Another powerful coded modulation scheme utilising bit-based - rather than symbol-based - channel interleaving in conjunction with Gray signal labeling is referred to as Bit-Interleaved Coded Modulation (BICM), which was proposed in $[6,7]$. It combines conventional convolutional codes with several independent bit interleavers, in order to increase the associated diversity order. In conjunction with bit interleavers, the code diversity order can be increased to the binary Hamming distance of a code, and the number of parallel bit-interleavers equals the number of coded bits in a symbol [6]. Recently, iteratively decoded BICM along with SP assisted signal labeling, referred to as BICM-ID has also been proposed [8-11]. The philosophy of BICM-ID is to increase the Euclidean distance of the BICM code and to exploit the full advan-

\begin{tabular}{|l|r|}
\hline Parameter & Value \\
\hline \hline Carrier Frequency & $1.9 \mathrm{GHz}$ \\
\hline Vehicular Speed & $30 \mathrm{mph}$ \\
\hline Doppler frequency & $85 \mathrm{~Hz}$ \\
\hline Normalised Doppler frequency & $3.25 \times 10^{-5}$ \\
\hline Channel type & C0ST 207 Typical Urban (see Figure 1) \\
\hline Number of paths in channel & 4 \\
\hline Data modulation & Adaptive Coded Modulation \\
& (4-QAM, 8-PSK, 16-QAM, 64-QAM) \\
\hline \multirow{2}{*}{ Receiver type } & Decision Feedback Equalizer \\
& Number of Forward Filter Taps = 35 \\
& Number of Backward Filter Taps = 7 \\
\hline
\end{tabular}

Table 1: Modulation and channel parameters

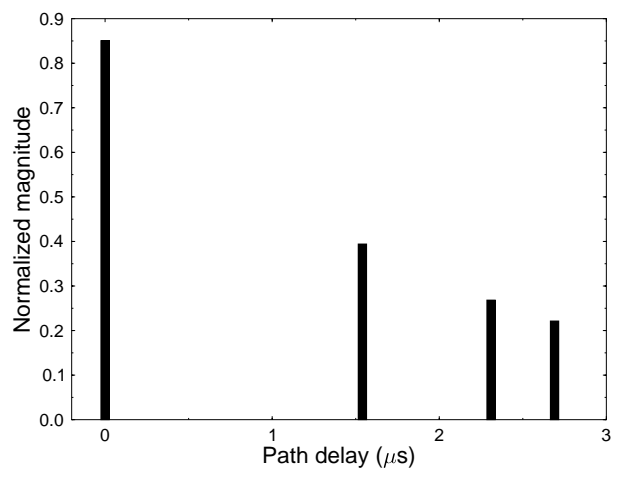

Figure 1: The impulse response of a COST 207 Typical Urban (TU) channel [12].

tage of bit interleaving upon invoking a simple iterative decoding technique.

\section{SYSTEM OVERVIEW}

Table 1 shows the modulation and channel parameters employed. The multi-path channel model is characterized by its discretised symbol-spaced COST207 Typical Urban (TU) channel impulse response [12], as shown in Figure 1, where each path is faded independently according to a Rayleigh distribution. The transmission burst structure of the FMA1 non-spread data specified in the FRAMES proposal [13], as shown in Figure 2, was used. The remaining system paramters are shown in Table 2.

The following assumptions are stipulated. Firstly, we assume that the equalizer is capable of estimating the Channel Impulse Response (CIR) perfectly from the equaliser training sequence of 


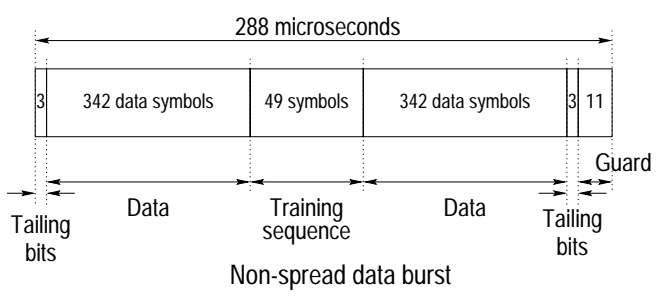

Figure 2: Transmission burst structure of the FMA1 non-spread data as specified in the FRAMES proposal [13].

\begin{tabular}{|l||r|}
\hline Features & Value \\
\hline \hline Multiple access & TDMA \\
\hline No. of Slots/Frame & 16 \\
\hline TDMA frame length & $4.615 \mathrm{~ms}$ \\
\hline TDMA slot length & $288 \mu \mathrm{s}$ \\
\hline Data Symbols/TDMA slot & 684 \\
\hline User Data Symbol Rate $(\mathrm{KBd})$ & 148.2 \\
\hline System Data Symbol Rate $(\mathrm{MBd})$ & 2.37 \\
\hline Symbols/TDMA slot & 750 \\
\hline User Symbol Rate $(\mathrm{KBd})$ & 162.5 \\
\hline System Symbol Rate $(\mathrm{MBd})$ & 2.6 \\
\hline System Bandwidth $(\mathrm{MHz})$ & 3.9 \\
\hline Eff. User Bandwidth $(\mathrm{kHz})$ & 244 \\
\hline
\end{tabular}

Table 2: Generic system features of the reconfigurable multi-mode video transceiver, using the non-spread data burst mode of the FRAMES proposal [13] shown in Figure 2.

Figure 2. Secondly, the CIR is time-invariant for the duration of a transmission burst, but varies from burst to burst according to the Doppler frequency, which corresponds to assuming that the CIR is slowly varying. The error propagation of the DFE will degrade the estimated performance, but the effect of error propagation is left for further study. The modulation modes that can be invoked by our multimode transceivers are 4QAM, 8PSK, 16QAM and 64QAM [14].

We invoke four encoders, each adding one parity bit to each information symbol, yielding the coding rate of $1 / 2$ in conjunction with the modulation mode of 4QAM, $2 / 3$ for $8 \mathrm{PSK}, 3 / 4$ for 16QAM and 5/6 for 64QAM. The TCM scheme invokes Ungerboeck's codes [1], while the TTCM scheme invokes Robertson's codes [4]. The BICM scheme was constructed using Paaske's convolutional codes [15]. The rate 5/6 code of the BICM scheme was constructed using Paaske's rate $1 / 2$ code and puncturing, following the approach of $[16,17]$. Soft decision trellis decoding utilizing the Log-Maximum A Posteriori (Log-MAP) algorithm [18] was invoked for the decoders. The Log-MAP algorithm is a numerically stable version of the MAP algorithm operating in the log-domain, in order to reduce its complexity and to mitigate the numerical problems associated with the MAP algorithm [19].

\section{VIDEO OVERVIEW}

The proposed video transceiver is based on the H.263 video codec [20]. The video coded bitstream is conveyed by an intelligent burst-by-burst adaptive coded modulation assisted wideband multimode modem [14], which can be configured either under network

\begin{tabular}{|l||c|c|c|c|c|}
\hline \multicolumn{1}{|l||}{ Features } & \multicolumn{4}{c|}{ Multi-rate System } \\
\hline \hline \multicolumn{1}{|l||}{ Mode } & 4QAM & 8PSK & 16QAM & 64QAM \\
\hline Transmission Symbols/TDMA slot & \multicolumn{5}{|c|}{684} \\
\hline Bits/Symbol & 2 & 3 & 4 & 6 \\
\hline Transmission bits/TDMA slot & 1368 & 2052 & 2736 & 4104 \\
\hline Packet Rate & \multicolumn{5}{|c|}{$216.7 / \mathrm{s}$} \\
\hline Transmission bitrate (kbit/s) & 296.4 & 444.6 & 592.8 & 889.3 \\
\hline Code Termination Symbols & \multicolumn{5}{|c|}{678} \\
\hline Data Symbols/TDMA slot & \multicolumn{5}{|c|}{16} \\
\hline Coding Rate & $1 / 2$ & $2 / 3$ & $3 / 4$ & $5 / 6$ \\
\hline Information Bits/Symbol & 1 & 2 & 3 & 5 \\
\hline Unprotected bits/TDMA slot & 678 & 1356 & 2034 & 3390 \\
\hline Unprotected bitrate (kbit/s) & 146.9 & 293.8 & 440.7 & 734.6 \\
\hline Video packet CRC (bits) & \multicolumn{5}{|c|}{9} \\
\hline Feedback protection (bits) & \multicolumn{5}{|c|}{30} \\
\hline Video packet header (bits) & 11 & 12 & 12 & 13 \\
\hline Video bits/packet & 642 & 1319 & 1997 & 3352 \\
\hline Effective Video-rate (kbit/s) & 139.1 & 285.8 & 432.7 & 726.3 \\
\hline Video framerate (Hz) & \multicolumn{5}{|c|}{30} \\
\hline
\end{tabular}

Table 3: Operational-mode specific transceiver parameters for TTCM

control or under transceiver control. This allowed us to support an increased throughput expressed in terms of the average number of bits per symbol, when the instantaneous channel quality was high, leading ultimately to an increased video quality in a constant bandwidth.

The video transceiver operates in close cooperation with the adaptive multi-mode modem, packetising the video bitstream into one packet per transmission burst. The number of bits in the video packet varies, depending on the current operating mode of the multi-mode modem. The video transceiver is informed of the packet's transmission success and failure by a highly protected feedback channel, which is integrated into the reverse link. More explicitly, the associated feedback flag is protected with the aid of a repetition code. The use of packet acknowledgement allows the video encoder and the remote decoder to operate on the basis of identical recontruction frame buffer contents without the need of retransmissions, which are wasteful in terms of bandwidth and delay.

The transmitted bitrate of all four modes of operation is shown in Table 3 for the TTCM scheme. The associated bitrates are similar for the other coded modulation schemes. The slight difference is caused by using different numbers of code termination symbols. The unprotected video bitrate before channel coding is also shown in the table. The actual useful bitrate available for video encoding is slightly lower, than the unprotected bitrate due to the bitrate allocation required by the strongly protected packet acknowledgement information, and packetisation overhead information. The effective video bitrate is also shown in the table, which varies from 139 to $726 \mathrm{kbit} / \mathrm{s}$.

We have investigated the video system concerned using a wide range of video sequences having different resolutions. However for consizeness we will only show results for the Common Intermediate Format (CIF) resolution (352x288 pixels) salesman sequence at 30 frames per second.

\section{EMPLOYING FIXED MODULATION MODES}

Initial simulations of the videophone transceiver were performed with the transceiver configured in one of the four fixed modulation modes of Table 3. We compare the performance of TTCM in 


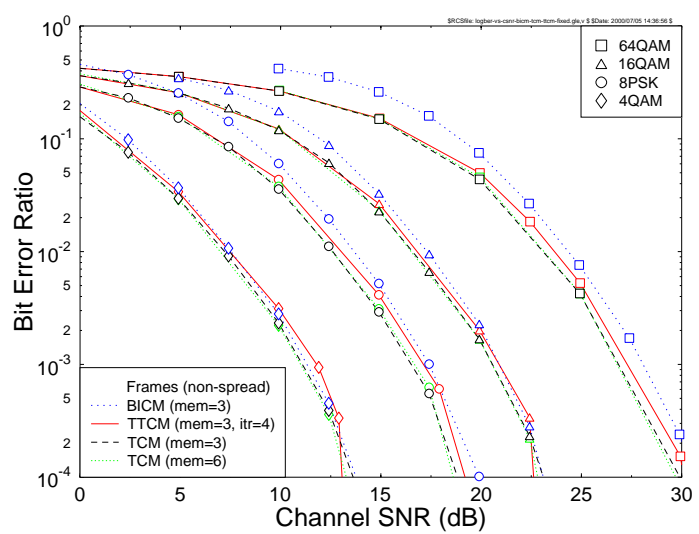

Figure 3: Bit-error-ratio versus channel SNR for the four fixed modem modes, using the four joint coding/modulation schemes considered, namely BICM, TCM3, TCM6 and TTCM over the channel of Figure 1.

conjunction with a constraint length of three and using four iterations, to that of non-iterative TCM along with a constraint length of six, since the associated computational complexity is similar. We also compare these results to TCM using a constraint length of three and to BICM employing a constraint length of three. Figure 3 shows the bit error ratio performance of the four different joint coding and modulation schemes for the four fixed modulation modes considered. It can be seen that the TCM schemes have the best performance. The BICM scheme has clearly the worst BER performance in the 8PSK, 16QAM and 64QAM modes.

Again, one video packet is transmitted in each TDMA frame and the receiver calculates, whether the received packet has any bit-errors using the associated Cyclic Redundancy Check (CRC). If the received packet has been corrupted, this acknowledgement message is strongly protected using repetition codes and superimposed on the reverse link transmission. In these investigations a transmission frame error resulted in a video packet error. We shall characterise the relative frequency of these events by the packet loss ratio (PLR). The PLR of the four joint coding and modulation schemes is shown in Figure 4.

Again, the BICM scheme has the worst performance. However, in terms of the PLR the TCM scheme using a constraint length of six has a significant performance advantage over the TCM scheme employing a constraint length of three. More noticeable is that the TTCM scheme provides the best PLR performance by a considerable margin. This is because turbo decoding of the TTCM scheme is very effective in reducing the number of bit errors to zero in packets exhibiting a low number of bit errors before decoding. The performance of BICM is worse, than that of TCM due to the associated limited channel interleaving depth [6,7] of the BICM scheme in our slow-fading wideband channels.

Figure 5 shows the error-free decoded video quality, measured in terms of the video peak signal to noise ratio (PSNR) versus time for the CIF resolution "Salesman" sequence for each of the four fixed modulation modes using the TTCM scheme. As expected, the figure demonstrates that the higher order modulation modes, which have a higher associated bitrate provide a better video quality. This graph will become useful at a later stage, when comparing the PSNR of the various scenarios obtained in an error-impaired

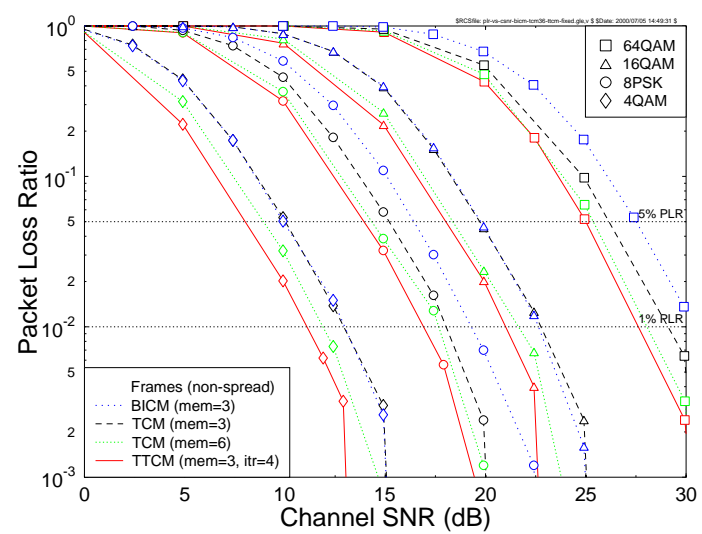

Figure 4: Packet loss ratio versus channel SNR for the four fixed modem modes, using the four joint coding/modulation schemes considered, namely BICM, TCM3, TCM6 and TTCM over the channel of Figure 1.

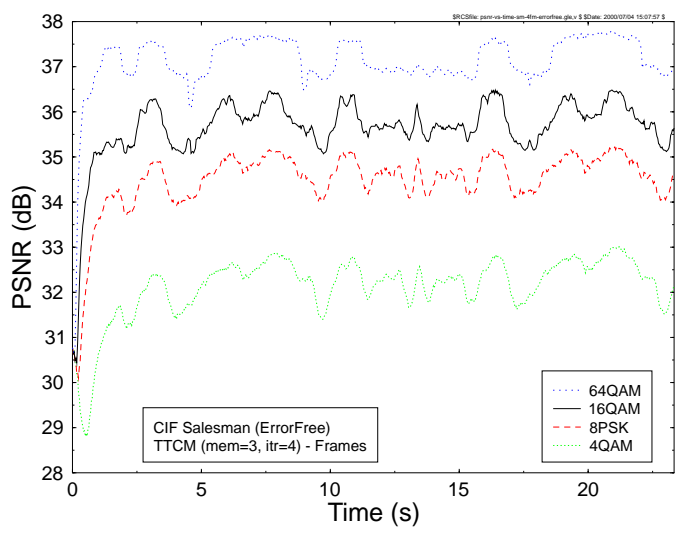

Figure 5: PSNR (video quality) versus time for the four fixed modulation modes, under error-free channel conditions using the CIF resolution "Salesman" video sequence at $30 \mathrm{frame} / \mathrm{s}$. Joint coding and modulation using turbo trellis coding with constraint length three and four iterations was employed.

situation.

\section{EMPLOYING TTCM/AQAM}

The modem mode switching mechanism of the adaptive schemes is characterised by a set of switching thresholds, by the corresponding random TTCM symbol-interleavers and the component codes, as follows:

Mod. Mode $= \begin{cases}4 Q A M, I_{0}=684, R_{0}=1 / 2 & \text { if } \gamma_{D F E} \leq f_{1} \\ 8 P S K, I_{1}=1368, R_{1}=2 / 3 & \text { if } f_{1}<\gamma_{D F E} \leq f_{2} \\ 16 Q A M, I_{2}=2052, R_{2}=3 / 4 & \text { if } f_{2}<\gamma_{D F E} \leq f_{3} \\ 64 Q A M, I_{3}=3420, R_{3}=5 / 6 & \text { if } \gamma_{D F E}>f_{3},\end{cases}$

where $f_{n}, n=1 . .3$ are the equalizer's output SNR thresholds, while $I_{n}$ represents the random TTCM symbol-interleaver size expressed in terms of the number of bits, which is not used for the TCM and 


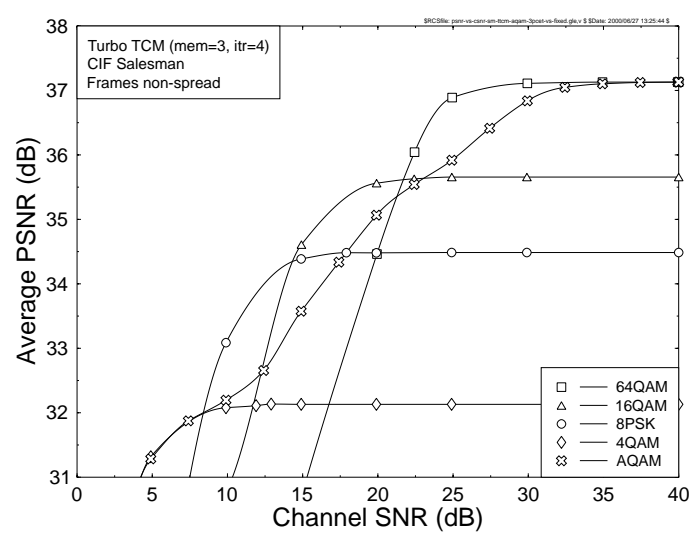

Figure 6: Average PSNR versus channel SNR for the four fixed TTCM modes and for the four-mode TTCM AQAM scheme using the CIF "Salesman" video sequence at 30 frame/s. A constraint length of three and four iterations were invoked over the COST207 CIR of Figure 1.

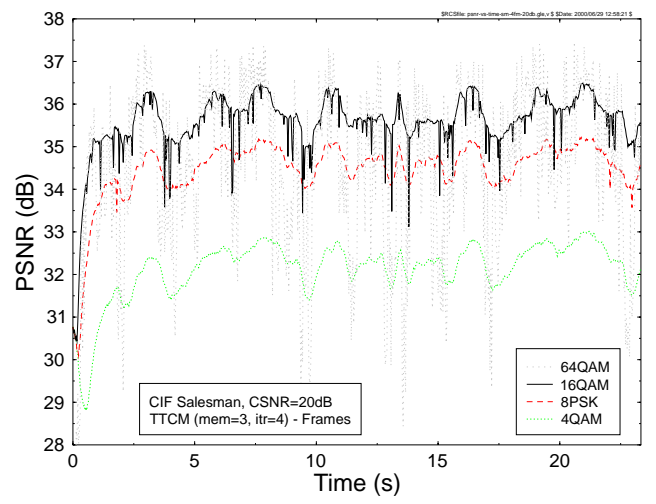

Figure 7: PSNR versus time for the four fixed modulation modes at a channel SNR of $20 \mathrm{~dB}$, using the CIF-resolution "Salesman" video sequence at 30 frames/s, employing a constraint length three and four iterations over the COST207 CIR of Figure 1.

BICM schemes. The switching thresholds $f_{n}$ were chosen using a heuristic algorithm, in order to maintain the required target PLR.

The video quality expressed in terms of the average PSNR is closely related to the effective throughput video bitrate, which is shown in Figure 6. As expected, the figure portrays that the AQAM modem's video performance degrades smoothly, as the channel SNR degrades, while the fixed modem's video performance degrades more rapidly, when the channel SNR becomes insufficient for the specific modem mode concerned. The video quality in terms of the PSNR is shown versus time in Figure 7 for the four fixed modulation modes at a channel SNR of $20 \mathrm{~dB}$. For further research on wireless video communications the interested reader is referred to [21].

\section{CONCLUSIONS}

In this contribution various burst-by-burst adaptive coded modulation based video transceivers have been studied. The near- instantaneously adaptive transceiver is capable of operating in four different coded modulation modes, namely 4QAM, 8PSK, 16QAM and 64QAM.

The advantage of using trellis coding in our near-instantaneously adaptive transceivers is that when invoking higher-order modulation modes in case of encountering a higher channel quality, the coding rate approaches unity. This allows us to maintain as high a throughput as possible. For example, the bitrate of the 4QAM and 64QAM modes was 296 and $889 \mathrm{Kbit} / \mathrm{s}$, repectively. The coding rate of the 4QAM mode was half, while that of the 64QAM mode was 5/6. This in turn allowed us to support effective video bitrates of 139 and 726Kbit/s in the 4QAM and 64QAM modes, repectively. The ratio of the effective video bitrate to the channel bitrate increased from $47 \%$ in the 4QAM mode to $82 \%$ in the 64QAM mode. We found that the turbo trellis coding scheme provided the best overall video performance due to its superior PLR performance.

The burst-by-burst adaptive modem guaranteed the same video performance as the lowest- and highest-order fixed-mode modulation schemes at extremely low and high channel SNRs, respectively. However in between these extreme SNRs the effective video bitrate smoothly increased, as the channel SNR increased, whilst maintaining a near-constant PLR. By controlling the AQAM switching thresholds a near-constant PLR can be maintained.

\section{REFERENCES}

[1] G. Ungerboeck, "Channel coding with multilevel/phase signals," IEEE Transactions on Information Theory, vol. IT-28, pp. 55-67, January 1982.

[2] D. Divsalar and M. K. Simon, "The design of trellis coded mpsk for fading channel: Performance criteria," IEEE Transactions on Communications, vol. 36, pp. 1004-1012, September 1988.

[3] D. Divsalar and M. K. Simon, "The design of trellis coded MPSK for fading channel: set partitioning for optimum code design," IEEE Transactions on Communications, vol. 36, pp. 1013-1021, September 1988.

[4] P. Robertson and T. Wörz, "Bandwidth efficient turbo trellis-coded modulation using punctured component codes," vol. 16, pp. 206-218, February 1998.

[5] C. Berrou, A. Glavieux, and P. Thitimajshima, "Near shannon limit error-correcting coding and decoding: Turbo codes," in Proceedings of the International Conference on Communications, (Geneva, Switzerland), pp. 1064-1070, May 1993.

[6] E. Zehavi, "8-PSK trellis codes for a Rayleigh fading channel," IEEE Transactions on Communications, vol. 40, pp. 873-883, May 1992.

[7] G. Caire, G. Taricco, and E. Biglieri, "Bit-interleaved coded modulation," IEEE Transactions on Information Theory, vol. 44, pp. 927-946, May 1998.

[8] X. Li and J. Ritcey, "Bit-interleaved coded modulation with iterative decoding," IEEE Communications Letters, vol. 1, November 1997.

[9] X. Li and J. Ritcey, "Bit-interleaved coded modulation with iterative decoding — approaching turbo-tcm performance 
without code concatenation," in Proceedings of CISS 1998, (Princeton University, USA), March 1998.

[10] X. Li and J. Ritcey, "Trellis-coded modulation with bit interleaving and iterative decoding," IEEE Journal on Selected Areas in Communications, vol. 17, April 1999.

[11] X. Li and J. Ritcey, "Bit-interleaved coded modulation with iterative decoding using soft feedback," IEE Electronics Letters, vol. 34, pp. 942-943, May 1998.

[12] "COST 207: Digital land mobile radio communications, final report." Office for Official Publications of the European Communities, 1989. Luxembourg.

[13] A. Klein, R. Pirhonen, J. Skoeld, and R. Suoranta, "FRAMES multiple access mode 1 - wideband TDMA with and without spreading," in Proceedings of IEEE International Symposium on Personal, Indoor and Mobile Radio Communications, PIMRC'97, vol. 1, (Marina Congress Centre, Helsinki, Finland), pp. 37-41, IEEE, 1-4 September 1997.

[14] L. Hanzo, W. Webb, and T. Keller, Single- and Multi-carrier Quadrature Amplitude Modulation. New York, USA: IEEE Press-John Wiley, April 2000.

[15] S. Lin and D. Constello Jr., Error Control Coding: Fundamentals and Applications. Englewood Cliffs, NJ, USA: Prentice-Hall, October 1982. ISBN: 013283796X.

[16] J. Hagenauer, "Rate-compatible puncture convolutional codes (RCPC) and their application," IEEE Transactions on Communications, vol. 36, pp. 389-400, April 1988.

[17] L. Lee, "New rate-compatible puncture convolutional codes for viterbi decoding," IEEE Transactions on Communications, vol. 42, pp. 3073-3079, December 1994.

[18] P. Robertson, E. Villebrun, and P. Hoeher, "A comparison of optimal and sub-optimal MAP decoding algorithms operating in the log domain," in Proceedings of the International Conference on Communications, pp. 1009-1013, June 1995.

[19] L. Bahl, J. Cocke, F. Jelinek, and J. Raviv, "Optimal decoding of linear codes for minimising symbol error rate," IEEE Transactions on Information Theory, vol. 20, pp. 284-287, March 1974.

[20] ITU-T, Recommendation H.263: Video Coding for Low Bitrate communication, March 1996.

[21] L. Hanzo, P. Cherriman, J. Streit: Wireless Video Communications: Second to Third Generation and Beyond, IEEE Press, 1

\footnotetext{
${ }^{1}$ For detailed contents please refer to http://wwwmobile.ecs.soton.ac.uk
} 